\title{
A Randomized Trial to Improve Adherence to Follow-up Eye Examinations Among People With Glaucoma
}

Benjamin E. Leiby, $\mathrm{PhD}^{1}$; Sarah E. Hegarty, MPhil ${ }^{1}$; Tingting Zhan, $\mathrm{PhD}^{1}$; Jonathan S. Myers, $\mathrm{MD}^{2,3}$;

L. Jay Katz, MD²,3 Julia A. Haller, MD ${ }^{3,4}$; Michael Waisbourd, MD5; Christine Burns ${ }^{2}$; Meskerem Divers ${ }^{2}$; Jeanne Molineaux ${ }^{2}$; Jeffrey Henderer, MD $^{6}$; Charles Brodowski, MD² Lisa A. Hark, PhD, RD ${ }^{2,7}$

\begin{abstract}
Accessible Version: www.cdc.gov/pcd/issues/2021/20_0567.htm Suggested citation for this article: Leiby BE, Hegarty SE, Zhan T, Myers JS, Katz LJ, Haller JA, et al. A Randomized Trial to Improve Adherence to Follow-up Eye Examinations Among People With Glaucoma. Prev Chronic Dis 2021;18:200567. DOI: https://doi.org/10.5888/pcd18.200567.
\end{abstract}

\section{PEER REVIEWED}

\section{Summary}

What is already known on this topic?

Nonadherence to follow-up eye care is common among people with glaucoma and other eye diseases. Use of patient navigators and social workers can increase adherence to eye care appointments.

What is added by this report?

The results of a randomized, controlled trial of patients with glaucoma and other eye diseases showed that a patient navigation and social work intervention doubled the rate of follow-up adherence in community settings.

What are the implications for public health practice?

Involving patient navigators and social workers in ophthalmic care could improve care and reduce disease progression.

\section{Abstract}

\section{Introduction}

Appointment nonadherence is common among people with glaucoma, making it difficult for eye care providers to monitor glaucoma progression. Our objective was to determine whether the use of patient navigators, in conjunction with social worker support, could increase adherence to recommended follow-up eye appointments.

\section{Results}

Timely attendance at the first visit was higher for EI than UC $(74.4 \%$ vs $39.0 \%$; average relative risk $[\mathrm{aRR}]=1.85 ; 95 \% \mathrm{CI}$, $1.51-2.28 ; P<.001)$. Rates of adherence to recommended annual follow-up during year 1 were $18.6 \%$ in the EI group and $8.1 \%$ in the usual care group (aRR $=2.08 ; 95 \% \mathrm{CI}, 1.14-3.76 ; P=.02)$. The aRR across years 2 and 3 was $3.92(95 \% \mathrm{CI}, 1.24-12.43 ; P=$ $.02)$.

\section{Conclusion}

An intervention using patient navigators and social workers doubled the rate of adherence to annual recommended follow-up eye care compared with usual care in community settings, and was effective at increasing connections with local ophthalmologists. Interventions to further improve long-term adherence are needed.

\section{Introduction}

Glaucoma is a chronic eye disease resulting in visual field defects and progressive vision loss and is the leading cause of irreversible blindness worldwide (1). Among other chronic diseases, diabetes in particular is associated with increased likelihood of developing 
glaucoma $(2,3)$. Because glaucoma is asymptomatic in early stages, early detection and timely intervention are critical to prevent vision loss (4). Nonadherence to recommended follow-up eye examinations reduces care and worsens outcomes (5). Fifty-four percent of people diagnosed with glaucoma fail to attend followup eye-related appointments (6). Barriers to nonadherence include health care costs, lack of transportation, and emotional distress $(7,8)$. These barriers most often affect people of color, who have lower attendance rates at follow-up eye care appointments than White patients (9-11).

Patient navigators and social workers can help address barriers to appointment adherence. Patient navigators direct patients to appropriate health care resources, coordinate and schedule appointments, verify insurance status, and arrange transportation $(12,13)$. Patient navigation programs have been used in other medical fields to promote adherence to medication and treatment (14-16). Few studies have looked at using patient navigators to improve appointment adherence among glaucoma patients, particularly among previously undiagnosed people with risk factors for glaucoma and eye disease $(12,17)$.

Social workers assess, track, and lessen psychosocial barriers to care to improve quality of life and patient well-being $(7,18)$. Social workers not only help patients navigate the health care system; they also provide emotional support, which has been shown to increase appointment adherence (18-20). In one study, a medical social worker in a pediatric ophthalmology setting increased appointment adherence by $45 \%(19,20)$. In several observational studies, glaucoma patients reported that a social worker resolved their issues and supported their keeping appointments with their ophthalmologist $(7,21)$.

The combined use of social workers and patient navigators to improve appointment adherence among glaucoma patients has not been investigated previously in a controlled, prospective adult study. Our objective was to determine whether the use of patient navigators and social workers could increase adherence to recommended follow-up eye appointments among a high-risk population with glaucoma or other eye diseases.

\section{Methods}

\section{Study design}

The Philadelphia Telemedicine Glaucoma Detection and Followup Study was a prospective, randomized clinical trial that aimed to address the issue of poor adherence to follow-up eye examinations by providing patient navigator and social worker support to directly guide participants through the eye care process (22). The 5 -year study was conducted by Wills Eye Hospital, funded by the
Centers for Disease Control and Prevention, and registered with ClinicalTrials.gov (NCT02390245). As described previously (22), the study's 2 phases 1) conducted a practice-based telemedicine screening program for glaucoma and other eye diseases among underserved populations with risk factors for eye disease and 2) evaluated whether a community intervention with patient navigation and social worker support improved access to and use of eye care. The study was approved by the Wills Eye Hospital Institutional Review Board and was conducted in accordance with the Declaration of Helsinki. Written informed consent was obtained from all participants before each phase of the study.

In Phase 1, the study aimed to identify people with undiagnosed glaucoma and other eye diseases and facilitate their referral to local ophthalmologists. A targeted sample at high risk for eye disease was recruited from 12 community partner organizations and consisted of African American, Hispanic, and Asian adults over age 40; adults over age 65 of any race/ethnicity; and people over age 40 with a family history of glaucoma or currently diagnosed with diabetes. We enrolled only people who had not seen an ophthalmologist in the previous 12 months $(\mathrm{N}=906)$. After informed consent was obtained, participants underwent a brief vision screening in their primary care provider's (PCP's) office (Visit 1), which included measuring visual acuity and intraocular pressure (IOP), and using fundus (retina) photography. Both retina and glaucoma specialists used telemedicine to read the images at Wills Eye Hospital. If the IOP was greater than $30 \mathrm{~mm} \mathrm{Hg}$, participants were immediately referred to a local ophthalmologist (fasttracked). Otherwise, participants with findings suggestive of sightthreatening disease, such as glaucoma, diabetic retinopathy, or hypertensive complications, or with unclear screening results were invited to return to the same location for a comprehensive eye examination by an ophthalmologist (Visit 2). At Visit 2, visual acuity and IOP were assessed again in addition to an ophthalmologic examination. Visual field tests were also performed, and visionrelated quality of life was assessed by using the National Eye Institute Vision Function Questionnaire (NEI-VFQ). Previous publications (22) report extensively on this first phase, including detailed methods and recruitment summary and concordance of the telemedicine eye screening findings and comprehensive examination diagnosis (23).

All participants who completed Visit 2 or who were fast-tracked were invited to participate in Phase 2 of the study. Phase 2 was a randomized controlled clinical trial designed to evaluate whether an enhanced intervention (EI) using patient navigation and social worker support improved patient adherence to follow-up eye care over usual care (UC) among those with newly diagnosed or suspected glaucoma or other ocular conditions.

The opinions expressed by authors contributing to this journal do not necessarily reflect the opinions of the U.S. Department of Health and Human Services, the Public Health Service, the Centers for Disease Control and Prevention, or the authors' affiliated institutions. 
Recruitment and randomization. Participants consenting to participate in Phase 2 were randomized to either the UC group or the EI group at a fixed 1:1 allocation ratio by using a masked method of random permuted blocks. Study coordinators retrieved the randomization and allowed participants to select an ophthalmologist they would like to follow up with over the next several years from a list of 20 participating offices located within 5 miles of the screening site.

Usual care. Participants randomized to UC were given their selected ophthalmologist's contact information and a copy of their eye examination results. UC participants were instructed to schedule an initial appointment with the ophthalmologist (Visit 3). Once connected to that ophthalmologist, services provided by each local ophthalmology practice generally included telephone calls and/ or text message reminders before appointments. No practices provided patient navigator or social worker assistance as part of their usual care during the study period.

Enhanced intervention. Participants randomized to EI received a team-based intervention that included comprehensive assessment by a licensed social worker and assistance from patient navigators. The social worker called EI participants up to 3 times within 2 weeks to conduct an initial assessment, explain the EI process, assess participants' understanding of their new or existing ocular diagnosis, and document current and past barriers to obtaining eye care. The social worker provided community resources for participants in need of food and medications at no cost or at a reduced cost and discussed options for transportation to the local ophthalmologist. The social worker also assessed the participant's ability to complete their activities of daily living and provided emotional support. EI participants interacted by telephone with the social worker at least 3 times per year over 2 years.

Wills Eye Hospital study managers, ocular technicians, and research assistants served as patient navigators for EI participants. Their responsibilities included calling participants to schedule appointments; confirming appointments by mail, email, and/or text messaging; arranging transportation through Customized Community Transportation and Philadelphia Paratransit Service; and scheduling language interpreters with medical training to participate in eye examinations as needed. Patient navigators were able to identify cultural and language differences and were aware of health literacy issues. When possible, navigators were race and language concordant with the patient population.

Management and follow-up examinations. At Visit 3 and each follow-up visit, the local ophthalmologist assessed the participant's ocular, medical, and family history and conducted a comprehensive eye examination based on their clinical practice.
The ophthalmologist would reconfirm ocular diagnoses, perform testing, adjust treatment recommendations as needed, and recommend follow-up intervals for the participant.

Final study visit. All randomized participants were invited to a final visit at their PCP's office at the end of the follow-up period. At this visit, the NEI-VFQ was re-administered, visual acuity and IOP were measured, and overall participant satisfaction with the study was assessed.

Outcome assessment. The research staff visited local ophthalmologists' offices to record visit dates, indications, findings, and treatments for up to 3 years from Visit 2. Data collection closed in March 2019.

Annual adherence. The primary outcome measure was adherence to recommended follow-up eye care appointments after Visit 3. Adherence was assessed annually on the basis of the expected follow-up schedule defined at the index visit for that year. In the first year, the follow-up recommendation given at Visit 3 by the ophthalmologist was classified into 1 of 4 categories: return within 2 months, return in 3 to 4 months, return in 6 months, or return in 12 months. This follow-up recommendation was then translated into the corresponding expected number of visits per year: 6 , 3,2 , or 1 . Participants were classified as adherent if the number of visits made within 13 months of Visit 3 (395 days) met or exceeded this expected number. Those who attended fewer visits or did not attend the initial visit with the local ophthalmologist within 12 months were deemed nonadherent for the first year. Adherence in the second and third years of follow-up was similarly defined; however, the follow-up recommendation used to define the required number of visits was based on the patient's most recent visit with the ophthalmologist before the start of that followup year. That is, the last visit that occurred during the first year of follow-up determined the follow-up schedule applied to the second year; similarly, the last visit that occurred during the second year of follow-up determined the follow-up schedule for the third year. When no visit occurred during a given year, the previous follow-up recommendation was carried forward. Additional measures of intervention effectiveness were explored, as detailed below.

Visit 3 attendance - initial visit with local ophthalmologist. The study evaluated the intervention's effectiveness in achieving the initial connection with the local ophthalmologist through timely attendance at Visit 3. Timely attendance was defined as having a first visit within 12 months of randomization.

Visit 4 attendance - first follow-up visit with local ophthalmologist. Adherence to the first follow-up visit (Visit 4) was assessed on the basis of the follow-up recommendation of the

The opinions expressed by authors contributing to this journal do not necessarily reflect the opinions of the U.S. Department of Health and Human Services, the Public Health Service, the Centers for Disease Control and Prevention, or the authors' affiliated institutions. 
local ophthalmologist at Visit 3. Participants with follow-up recommended within 2 months were deemed adherent to the first follow-up visit if they returned within 3 months; for recommended follow-up of 3 to 4 months, 6 months, or 12 months, patients were considered adherent to Visit 4 within 6,12 , or 15 months, respectively.

Total number of visits with local ophthalmologist. The total number of visits included all visits on distinct days occurring after randomization, including Visit 3.

Satisfaction. A brief questionnaire was administered at the final study visit to assess overall satisfaction. By using a 4-point Likerttype scale, participants were asked to state their satisfaction with the study and the local ophthalmologist, perceived helpfulness of the study toward understanding their recommended eye-care, and likeliness to continue with follow-up care at the local ophthalmologist.

\section{Statistical analysis}

The study was designed to detect a $20 \%$ difference in adherence rates between groups during the first year of follow-up by using a 2-tailed test with $\alpha=0.025$. With a final sample size of at least 135 participants per group, power to detect such a difference was $86 \%$ when the overall adherence across both study arms was $50 \%$.

Participant characteristics at Visits 1 and 2 were summarized by randomization arm by using means and SDs or number and percentages. Adjusted estimates of the relative risk (aRR) of timely attendance at Visit 3 were calculated by using Poisson regression in a generalized estimating equation framework (24). An extension of this model for longitudinal data was used to jointly model repeated annual measures of follow-up adherence (25). The longitudinal model included time (year 1, 2, or 3), randomization assignment, and randomization by time interaction. Both models adjusted for baseline characteristics believed to be associated with adherence to follow-up: Visit 2 recommended follow-up (as a surrogate for disease severity), age at screening, sex, insurance type, and baseline NEI-VFQ composite score.

In the longitudinal model, 2 relative risks were calculated and 2 hypotheses were tested: 1) comparing randomization groups at year 1 to assess differences in early adherence and 2) calculating the average effect of randomization group across years 2 and 3 to test the long-term efficacy of the intervention. Each test was performed with $\alpha=0.025$. Supporting analyses compared groups with respect to the percentage of participants who attended any visits with the local ophthalmologist, the percentage of participants who were adherent to their first post-Visit 3 visit, and the total number of visits after randomization. Analyses of dichotomous end points used the same approach as Visit 3 analysis. Number of visits was modeled by Poisson regression with follow-up time from randomization as the offset. An exploratory subset analysis was performed for participants with glaucoma-related diagnoses (those diagnosed with glaucoma, glaucoma suspect, or ocular hypertension). All analyses were performed by using SAS 9.4 and SAS/ STAT 14.3 (SAS Institute).

\section{Results}

\section{Participant characteristics}

From April 2015 through February 2017, 906 participants completed the telemedicine eye vision screening (Visit 1) with their PCP as part of Phase 1 of this 5-year study (Figure 1) (22). On telemedicine reading, 355 participants $(39 \%)$ were classified as having normal fundus images. The remaining 551 participants had abnormal or suspicious fundus images (334, 37\%), unreadable fundus images $(155,17 \%)$, or IOP exceeding $21 \mathrm{~mm} \mathrm{Hg}(62,7 \%)$. Fifteen participants had IOP $>30 \mathrm{~mm} \mathrm{Hg}$ that required fast-track referral to the local ophthalmologist. The other 536 participants were invited to have a comprehensive eye examination by their PCP; 347 participants (65\%) attended this Visit 2. These 347 patients and the 15 fast-tracked patients were invited to participate in Phase 2. A total of 344 participants consented and were randomized to either EU $(n=172)$ or UC $(n=172)$. Participants were followed up for a minimum of 22 months post-randomization.

The opinions expressed by authors contributing to this journal do not necessarily reflect the opinions of the U.S. Department of Health and Human Services, the Public Health Service, the Centers for Disease Control and Prevention, or the authors' affiliated institutions. 


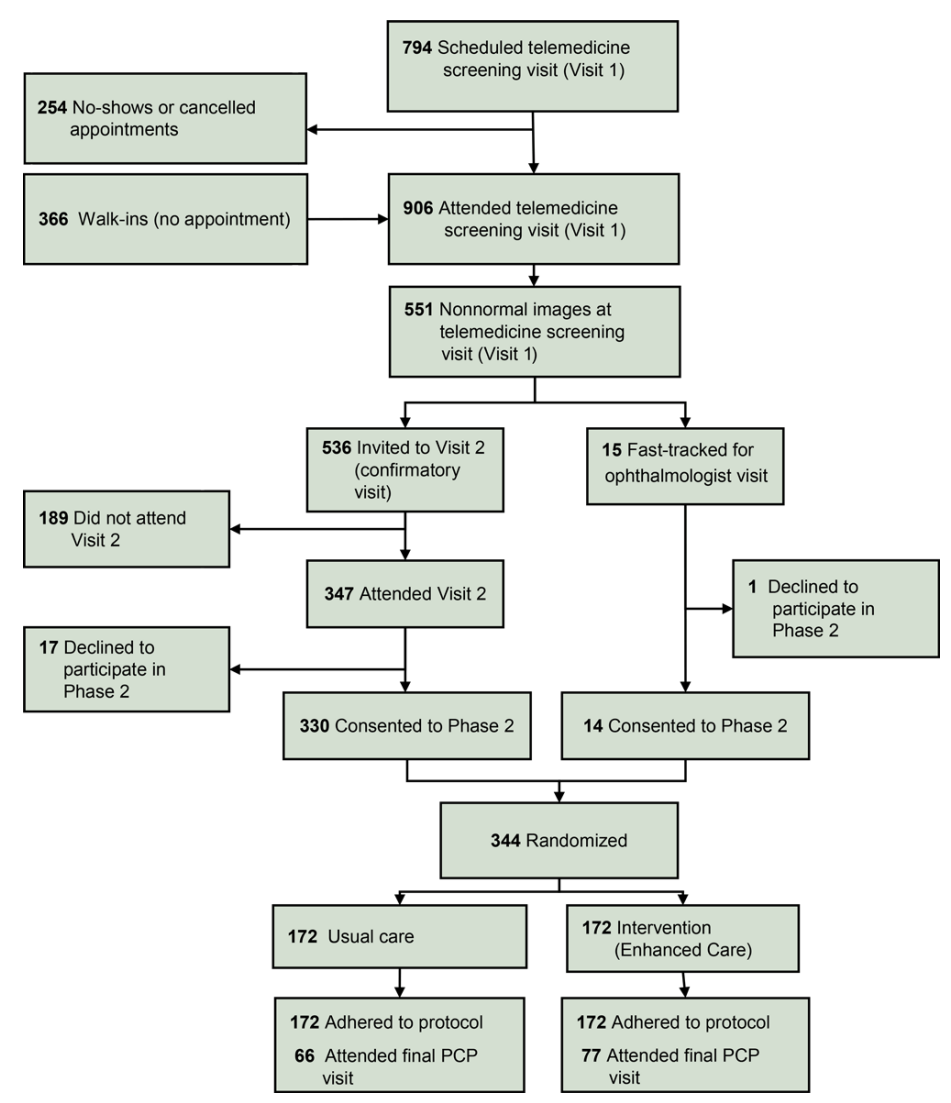

Figure 1. Flow chart describing the Philadelphia Telemedicine Glaucoma Detection and Follow-up Study, indicating participant inclusion, exclusion, and randomization to the usual care group or enhanced intervention group.

The mean age of participants was 59.9 years at screening; most (59\%) were women and 66\% were African American (Table 1). Roughly two-thirds $(\mathrm{n}=230,66.9 \%)$ had a glaucoma-related diagnosis at Visit 2 or were fast-tracked to visit an ophthalmologist because of high IOP. An NEI-VFQ average composite score of 82 indicated somewhat diminished vision-related quality of life. We saw no large differences in randomization groups with respect to baseline characteristics, although the EI group had a slightly higher percentage of women and a lower percentage of participants with diabetes (Table 1).

Timely Visit 3 attendance. About half of participants (56.7\% $[74.4 \% \mathrm{EI}, 39.0 \% \mathrm{UC}]$ ) attended the initial visit with the local ophthalmologist within 12 months of randomization (Table 2). In adjusted analysis (Table 2), the EI group showed an $85 \%$ relative increase in timely Visit 3 attendance (adjusted relative risk [aRR] $=1.85 ; 95 \% \mathrm{CI}, 1.51-2.28 ; P<.001)$. The effect was similar in the subset of participants with glaucoma-related diagnoses $(\mathrm{aRR}=$ $1.73 ; 95 \%$ CI $1.37-2.19 ; P<.001)$. Among those who made timely contact with the local ophthalmologist, the median time to first visit was 57 days (interquartile range [IQR]: 39-92) in EI and 47 days (IQR: 27-82) in UC. Rates of any attendance at the local ophthalmologist were also higher in EI (77.9\% vs $41.3 \%$; aRR = 1.83 ; 95\% CI, 1.51-2.22; Table 2) although only 6 EI and 4 UC participants ever attended a later visit after failing to make contact with the ophthalmologist in the first 12 months.

Adherence to follow-up after Visit 3. In year 1, the adherence rate was $18.6 \%$ in the EI group and $8.1 \%$ in UC with an aRR of 2.08 (95\% CI, 1.14-3.76; $P=.016$ ) indicating that the intervention significantly increased the rate of adherence (Figure 2) (Table 3). Adherence was relatively stable in years 2 and 3 for EI, while declining over time in UC. The average aRR of adherence across years 2 and 3 was $3.92(95 \% \mathrm{CI}, 1.24-12.43 ; P=.02)$. Results were similar in an exploratory analysis of the glaucoma-related diagnosis subset with an aRR of 2.30 for year 1 adherence $(95 \% \mathrm{CI}$, $1.10-4.82)$ and 3.44 across years 2 and 3 (95\% CI, 1.11-10.63) (Table 3).

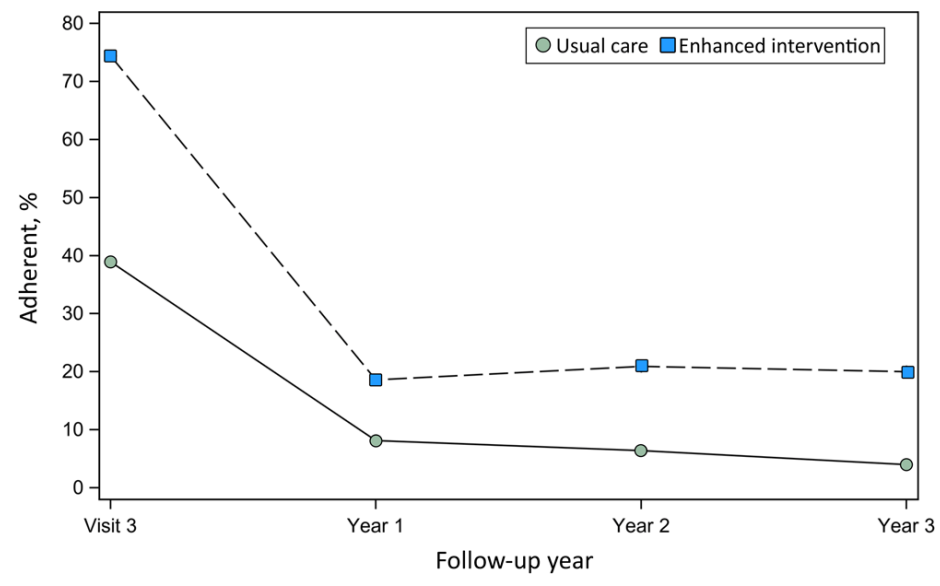

Figure 2. Adherence to recommended follow-up schedule over time by intervention group. Visit 3 was the initial visit with the community ophthalmologist. Timely adherence to Visit 3 was defined as attendance within 12 months of randomization. Annual adherence in Years 1-3 was defined as having attended all recommended follow-up visits within 13 months based on the recommended follow-up at the visit closest to the beginning of the year.

For adherence to the first follow-up visit recommended by the local ophthalmologist (Visit 4), the rate was $56.4 \%$ for EI group and $22.7 \%$ for $\mathrm{UC}$ (aRR $=2.39$; 95\% CI, 1.78-3.22) (Table 2). The average number of visits per year of follow-up was 0.9 in EI and 0.4 in $\mathrm{UC}(\mathrm{aRR}=2.07 ; 95 \% \mathrm{CI}, 1.54-2.78)$ (Table 2). The proportion of participants who attended at least 1 visit in the first year of follow-up was $41.3 \%$ in $\mathrm{EI}$ and $18.0 \%$ in $\mathrm{UC}(\mathrm{aRR}=2.18 ; 95 \%$ CI, 1.52-3.12) (Table 2).

Final study visit and satisfaction survey. One-hundred forty-three participants attended the final study visit at the PCP office (EI, 77; 
UC, 66). Both groups were satisfied or very satisfied with participation in the study (EI, 98.7\%; UC, 93.9\%), and most participants in both groups found the study very helpful in understanding and taking care of their eyes (EI, 64.9\%; UC, 54.5\%).

\section{Discussion}

In analysis of our primary outcome, we found that an intervention combining the support of patient navigators and social workers doubled adherence to recommendations for follow-up with a local ophthalmologist during the first year. These effects were similar for participants with or without glaucoma-related diagnoses. Much of this effect was likely due to an $85 \%$ relative increase in timely attendance at the initial visit with the ophthalmologist (Visit 3) for those randomized to the intervention arm (EI). After the first year, adherence rates dropped, but were still higher in the EI group.

Our results are similar to previous studies. The UC group in our study had only $39 \%$ attendance at the initial ophthalmologist visit, similar to results from the Hoffberger program, which provided free community-based eye screenings to residents of Baltimore, Maryland, at high risk for eye disease (6). In another prospective study, after 1 year, participants with glaucoma-related diagnoses had $82.5 \%$ follow-up adherence rates with the help of only patient navigators in office-based settings, compared with $73.3 \%$ in the usual care group; however, differences were not significant (12). Adherence in this study was defined as 1 or more visits within 1 year of diagnosis, and these rates were similar to what we observed for the same outcome in our intervention group (74.4\%). Although the UC rate was much lower in our study, it is consistent with low rates seen in another recent study (26).

Patients with glaucoma may face barriers to receiving follow-up eye care, which should be recognized and addressed. A questionnaire presented to patients in a glaucoma clinic who were referred to a medical social worker found that the most frequent barrier to receiving eye care was emotional distress; additional barriers were cost of visits, lack of insurance, transportation, impairment of daily activities, and language (7). Another study reported forgetfulness as a major barrier to adherence to follow-up care (27). Degree of depression was also correlated with level of nonadherence to eye care recommendations (28). The results of our study suggest that combining support of patient navigators and social workers may be effective in reducing these barriers and thereby improving outcomes.

Our low annual adherence rates may be because adherence for the year was defined on the basis of the follow-up recommendation at the beginning of the year. For example, if a participant was given a recommendation during Visit 3 to follow up in 2 months, this was considered the desired follow-up interval throughout the following year. However, recommendations for follow-up could have varied during the year, and this may have affected our annual adherence results.

Our study had several limitations. First, in spite of the improved adherence in the EI, annual adherence was still unacceptably low compared to what is necessary for adequate treatment. Second, our sample size for year 3 limited our ability to assess the long-term benefit of the intervention. Lastly, different ophthalmologists' offices used diverse measures to remind patients to return for follow-up eye examinations, which were not controlled and could have affected our results.

This study targeted a diverse, urban population at risk for glaucoma but not receiving regular eye care. Study results would likely be generalizable to similar settings, although access to care, insurance rates, and existing support systems are likely to differ in other geographic areas and may affect the benefit of the intervention.

Future studies could consider combining additional interventions to further increase rates of adherence to follow-up eye care. Other interventions that may show promise include providing incentives such as free eyeglass prescriptions or free eyeglasses (26) and providing other financial incentives to encourage at-risk participants to return for follow-up eye examinations (12). The costs of the screening phase have been previously reported (22); the costeffectiveness of our adherence intervention is being evaluated. In conclusion, our study addresses a critical gap in ophthalmic care by improving adherence to follow-up recommendations by using patient navigators and social workers. Addressing this gap is important because adherence to eye care contributes to a better prognosis for patients with chronic eye disease. We believe that use of social workers and patient navigators could be scaled on a national level to decrease the growing burden associated with glaucoma and other sight-threatening eye diseases.

\section{Acknowledgments}

Research for this article was supported by cooperative agreement U01 DP005127 from the Vision Health Initiative, Division of Diabetes Translation, National Center for Chronic Disease Prevention and Health Promotion, Centers for Disease Control and Prevention.

The following authors have financial disclosures:

Jonathan S. Myers received grants or research support from Aerie Pharmaceuticals, Inc, Allergan, Diopsys, Glaukos Corporation, Heidelberg Engineering, and Zeiss US. He has served as a consult-

\footnotetext{
The opinions expressed by authors contributing to this journal do not necessarily reflect the opinions of the U.S. Department of Health and Human Services, the Public Health Service, the Centers for Disease Control and Prevention, or the authors' affiliated institutions.
} 
ant for Aerie, Allergan, Glaukos, and MicrOptx and as a speaker for Aerie Pharmaceuticals, Inc, and Allergan.

Julia Haller has served as a consultant for KalVista Pharmaceuticals, Lowy Medical Research Institute, Merck, Novartis Pharmaceuticals, and Spark Therapeutics. She is a member of the board of directors of Bristol Myers Squibb Company and a member of the data safety monitoring boards of Aura Biosciences, Janssen Pharmaceuticals, and Lowy Medical Research Institute. She is an independent contractor for Alcon.

L. Jay Katz received grants or research support from Heidelberg Engineering, Inc, and Zeiss. He has served as a consultant and advisory board member for Allergan and Bausch and Lomb, Inc, and is on the speakers list for Allergan, Glaukos, and Bausch and Lomb, Inc. He holds stock in Glaukos, Mati Therapeutics, Aerie Pharmaceuticals, Inc, and Olleyes, Inc. He is chief medical officer of Glaukos.

Michael Waisbourd received research support from Novartis Pharmaceuticals. He is a consultant for IOPtima Ltd and a speaker for Novartis Pharmaceuticals.

Lisa Hark received funding from the Centers for Disease Control and Prevention Vision Health Initiative for the Manhattan Vision Screening and Follow-up Study in Vulnerable Populations and the Coordinating Center for Community Based-Interventions with Vulnerable Populations.

The findings and conclusions in this article are those of the authors and do not necessarily represent the official position of the Centers for Disease Control and Prevention. No copyrighted material was used in this article.

\section{Author Information}

Corresponding Author: Jonathan S. Myers, MD, Glaucoma Service, Wills Eye Hospital, 840 Walnut St, Ste 1110 , Philadelphia PA 19107. Telephone: 215-928-3197. Email: JMyers@willseye.org.

Author Affiliations: ${ }^{1}$ Thomas Jefferson University, Sidney Kimmel Medical College, Department of Pharmacology and Experimental Therapeutics, Division of Biostatistics, Philadelphia, Pennsylvania. ${ }^{2}$ Wills Eye Hospital, Glaucoma Research Center, Philadelphia, Pennsylvania. ${ }^{3}$ Thomas Jefferson University, Sidney Kimmel Medical College, Department of Ophthalmology, Philadelphia, Pennsylvania. ${ }^{4}$ Wills Eye Hospital, Office of the Ophthalmologist-in-Chief, Philadelphia, Pennsylvania. ${ }^{5}$ Department of Ophthalmology, Tel Aviv Medical Center, affiliated with the Sackler Faculty of Medicine, Tel Aviv
University, Israel. ${ }^{6}$ Temple University School of Medicine, Department of Ophthalmology, Philadelphia, Pennsylvania. ${ }^{7}$ Columbia University Irving Medical Center, Department of Ophthalmology, New York, New York.

\section{References}

1. Quigley HA, Broman AT. The number of people with glaucoma worldwide in 2010 and 2020. Br J Ophthalmol 2006; 90(3):262-7.

2. Chopra V, Varma R, Francis BA, Wu J, Torres M, Azen SP; Los Angeles Latino Eye Study Group. Type 2 diabetes mellitus and the risk of open-angle glaucoma the Los Angeles Latino Eye Study. Ophthalmology 2008;115(2):227-232.e1.

3. Pasquale LR, Kang JH, Manson JE, Willett WC, Rosner BA, Hankinson SE. Prospective study of type 2 diabetes mellitus and risk of primary open-angle glaucoma in women. Ophthalmology 2006;113(7):1081-6.

4. Tham YC, Li X, Wong TY, Quigley HA, Aung T, Cheng CY. Global prevalence of glaucoma and projections of glaucoma burden through 2040: a systematic review and meta-analysis. Ophthalmology 2014;121(11):2081-90.

5. Ung C, Murakami Y, Zhang E, Alfaro T, Zhang M, Seider MI, et al. The association between compliance with recommended follow-up and glaucomatous disease severity in a county hospital population. Am J Ophthalmol 2013;156(2):362-9.

6. Quigley HA, Park CK, Tracey PA, Pollack IP. Community screening for eye disease by laypersons: the Hoffberger program. Am J Ophthalmol 2002;133(3):386-92.

7. Fudemberg SJ, Amarasekera DC, Silverstein MH, Linder KM, Heffner P, Hark LA, et al. Overcoming barriers to eye care: patient response to a medical social worker in a glaucoma service. J Community Health 2016;41(4):845-9.

8. Sleath B, Blalock SJ, Carpenter DM, Sayner R, Muir KW, Slota C, et al. Ophthalmologist-patient communication, selfefficacy, and glaucoma medication adherence. Ophthalmology 2015;122(4):748-54.

9. Fudemberg SJ, Lee B, Waisbourd M, Murphy RA, Dai Y, Leiby BE, et al. Factors contributing to nonadherence to follow-up appointments in a resident glaucoma clinic versus primary eye care clinic. Patient Prefer Adherence 2016; 10:19-25.

10. Lee BW, Murakami Y, Duncan MT, Kao AA, Huang JY, Lin $\mathrm{S}$, et al. Patient-related and system-related barriers to glaucoma follow-up in a county hospital population. Invest Ophthalmol Vis Sci 2013;54(10):6542-8.

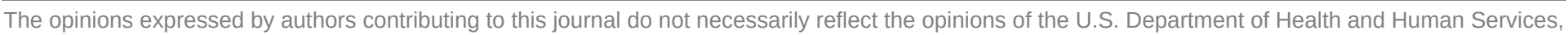
the Public Health Service, the Centers for Disease Control and Prevention, or the authors' affiliated institutions. 
11. Zhang X, Cotch MF, Ryskulova A, Primo SA, Nair P, Chou $\mathrm{CF}$, et al. Vision health disparities in the United States by race/ ethnicity, education, and economic status: findings from two nationally representative surveys. Am J Ophthalmol 2012; 154(6Suppl):S53-62.e1, S62.e1.

12. Zeng L, Hark LA, Johnson DM, Berardi G, Patel NS, Dai Y, et al. A randomized, controlled trial to test the effectiveness of a glaucoma patient navigator to improve appointment adherence. Patient Prefer Adherence 2016;10:1739-48.

13. Mitrowski CA. Patient advocate: the role of the medical social worker. Prog Clin Biol Res 1983;121:273-8.

14. Freund KM, Battaglia TA, Calhoun E, Darnell JS, Dudley DJ, Fiscella K, et al.; Writing Group of the Patient Navigation Research Program. Impact of patient navigation on timely cancer care: the Patient Navigation Research Program. J Natl Cancer Inst 2014;106(6):dju115.

15. Raich PC, Whitley EM, Thorland W, Valverde P, Fairclough D; Denver Patient Navigation Research Program. Patient navigation improves cancer diagnostic resolution: an individually randomized clinical trial in an underserved population. Cancer Epidemiol Biomarkers Prev 2012; 21(10):1629-38.

16. Honeycutt S, Green R, Ballard D, Hermstad A, Brueder A, Haardörfer R, et al. Evaluation of a patient navigation program to promote colorectal cancer screening in rural Georgia, USA. Cancer 2013;119(16):3059-66.

17. Hark L, Waisbourd M, Myers JS, Henderer J, Crews JE, Saaddine JB, et al. Improving access to eye care among persons at high-risk of glaucoma in Philadelphia - design and methodology: the Philadelphia Glaucoma Detection and Treatment Project. Ophthalmic Epidemiol 2016;23(2):122-30.

18. Miller JJ, Frost MH, Rummans TA, Huschka M, Atherton P, Brown $\mathrm{P}$, et al. Role of a medical social worker in improving quality of life for patients with advanced cancer with a structured multidisciplinary intervention. J Psychosoc Oncol 2007;25(4):105-19.

19. Dotan G, Truong B, Snitzer M, McCauley C, MartinezHelfman S, Santa Maria K, et al. Outcomes of an inner-city vision outreach program: give kids sight day. JAMA Ophthalmol 2015;133(5):527-32.

20. Pizzi LT, Snitzer M, Amos T, Prioli KM, Steele D, Levin AV. Cost and effectiveness of an eye care adherence program for Philadelphia children with significant visual impairment. Popul Health Manag 2015;18(3):223-31.

21. Hark LA, Madhava M, Radakrishnan A, Anderson-Quiñones C, Robinson D, Adeghate J, et al. Impact of a social worker in a glaucoma eye care service: a prospective study. Health Soc Work 2019;44(1):48-56.
22. Hark LA, Katz LJ, Myers JS, Waisbourd M, Johnson D, Pizzi LT, et al. Philadelphia Telemedicine Glaucoma Detection and Follow-up Study: methods and screening results. Am J Ophthalmol 2017;181:114-24.

23. Hark LA, Myers JS, Ines A, Jiang A, Rahmatnejad K, Zhan T, et al. Philadelphia Telemedicine Glaucoma Detection and Follow-up Study: confirmation between eye screening and comprehensive eye examination diagnoses. Br J Ophthalmol 2019;103(12):1820-6.

24. Zou G. A modified poisson regression approach to prospective studies with binary data. Am J Epidemiol 2004;159(7):702-6.

25.Zou GY, Donner A. Extension of the modified Poisson regression model to prospective studies with correlated binary data. Stat Methods Med Res 2013;22(6):661-70.

26. Zhao D, Guallar E, Bowie JV, Swenor B, Gajwani P, Kanwar $\mathrm{N}$, et al. Improving follow-up and reducing barriers for eye screenings in communities: the SToP Glaucoma Study. Am J Ophthalmol 2018;188:19-28.

27. Zheng CX, Hu WD, Tran J, Siam L, Berardi GG, Sembhi H, et al. Barriers to receiving follow-up eye care and detection of non-glaucomatous ocular pathology in the Philadelphia Glaucoma Detection and Treatment Project. J Community Health 2016;41(2):359-67.

28. Weiss GA, Goldich Y, Bartov E, Burgansky-Eliash Z. Compliance with eye care in glaucoma patients with comorbid depression. Isr Med Assoc J 2011;13(12):730-4.

The opinions expressed by authors contributing to this journal do not necessarily reflect the opinions of the U.S. Department of Health and Human Services, the Public Health Service, the Centers for Disease Control and Prevention, or the authors' affiliated institutions. 


\section{Tables}

Table 1. Demographic and Clinical Characteristics of Subjects $(\mathrm{N}=344)$ Randomized to Usual Care and Intervention Groups, the Philadelphia Telemedicine Glaucoma Detection and Follow-up Study

\begin{tabular}{|c|c|c|c|}
\hline Characteristic & All $(N=344)$ & Usual Care $(n=172)$ & Intervention $(n=172)$ \\
\hline Age, y, mean (SD) & $59.9(11.0)$ & $59.0(10.6)$ & $60.8(11.4)$ \\
\hline \multicolumn{4}{|l|}{ Sex, n (\%) } \\
\hline Female & $202(58.7)$ & $94(54.7)$ & $108(62.8)$ \\
\hline Male & $142(41.3)$ & $78(45.3)$ & $64(37.2)$ \\
\hline \multicolumn{4}{|l|}{ Race/ethnicity ${ }^{\mathrm{a}}, \mathrm{n}(\%)$} \\
\hline African American & $223(66.2)$ & $111(66.1)$ & $112(66.3)$ \\
\hline White & $52(15.4)$ & $25(14.9)$ & $27(16.0)$ \\
\hline Asian & $16(4.8)$ & $7(4.2)$ & $9(5.3)$ \\
\hline Hispanic & $37(11.0)$ & $20(11.9)$ & $17(10.1)$ \\
\hline More than one race & $9(2.7)$ & $5(3.0)$ & $4(2.4)$ \\
\hline Family of history glaucoma, $\mathrm{n}(\%)$ & $87(25.3)$ & $49(28.5)$ & $38(22.1)$ \\
\hline Current smoker, n (\%) & $95(27.6)$ & $45(26.2)$ & $50(29.1)$ \\
\hline Hypertension, n (\%) & $237(68.9)$ & $122(70.9)$ & $115(66.9)$ \\
\hline Diabetes, n (\%) & $198(57.6)$ & $108(62.8)$ & $90(52.3)$ \\
\hline \multicolumn{4}{|l|}{ Insurance type, $\mathrm{n}(\%)$} \\
\hline Medicaid & $130(37.8)$ & $64(37.2)$ & $66(38.4)$ \\
\hline Medicare & $91(26.5)$ & $40(23.3)$ & $51(29.7)$ \\
\hline Private & $99(28.8)$ & $54(31.4)$ & $45(26.2)$ \\
\hline None & $24(7.0)$ & $14(8.1)$ & $10(5.8)$ \\
\hline \multicolumn{4}{|l|}{ Screening outcome, $n$ (\%) } \\
\hline Abnormal & $218(63.4)$ & $112(65.1)$ & $106(61.6)$ \\
\hline Unreadable & $85(24.7)$ & $38(22.1)$ & $47(27.3)$ \\
\hline Ocular hypertension & $41(11.9)$ & $22(12.8)$ & $19(11.0)$ \\
\hline \multicolumn{4}{|l|}{ Visit 2 recommended follow-up, $n$ (\%) } \\
\hline Every 3-4 months & $54(15.7)$ & $26(15.1)$ & $28(16.3)$ \\
\hline Every 6 months & $115(33.4)$ & $59(34.3)$ & $56(32.6)$ \\
\hline Every 12 months & $175(50.9)$ & $87(50.6)$ & $88(51.2)$ \\
\hline \multicolumn{4}{|l|}{ logMAR visual, mean (SD) } \\
\hline Lower (better) & $0.2(0.2)$ & $0.2(0.2)$ & $0.2(0.2)$ \\
\hline Higher (worse) & $0.3(0.4)$ & $0.3(0.5)$ & $0.3(0.3)$ \\
\hline
\end{tabular}

Abbreviations: C/D ratio, cup-to-disc ratio; dB, decibel; IOP, intraocular pressure; logMAR, logarithm of the minimum angle of resolution; NEI-VFQ, National Eye Institute Visual Function Questionnaire.

${ }^{a}$ Race was unknown for 7 subjects.

${ }^{b}$ IOP was carried forward from visit 1 for 17 subjects (including 14 fast-tracked subjects).

${ }^{\mathrm{C}} \mathrm{C} / \mathrm{D}$ ratio was not available for 22 subjects (including 14 fast-tracked subjects).

${ }^{\mathrm{d}}$ Mean deviation was not available for 19 subjects (including 14 fast-tracked subjects).

${ }^{\mathrm{e}}$ One subject did not complete the questionnaire; samples sizes vary across subscales.

(continued on next page)

The opinions expressed by authors contributing to this journal do not necessarily reflect the opinions of the U.S. Department of Health and Human Services, the Public Health Service, the Centers for Disease Control and Prevention, or the authors' affiliated institutions. 
(continued)

Table 1. Demographic and Clinical Characteristics of Subjects $(\mathrm{N}=344)$ Randomized to Usual Care and Intervention Groups, the Philadelphia Telemedicine Glaucoma Detection and Follow-up Study

\begin{tabular}{|c|c|c|c|}
\hline Characteristic & All $(N=344)$ & Usual Care $(n=172)$ & Intervention $(n=172)$ \\
\hline \multicolumn{4}{|l|}{$\mathrm{IOP}^{\mathrm{b}}, \mathrm{mmHg}$, mean (SD) } \\
\hline Lower (better) & $14.9(4.4)$ & $15.3(4.7)$ & $14.5(4.0)$ \\
\hline Higher (worse) & $16.6(5.2)$ & $16.9(5.3)$ & $16.2(5.0)$ \\
\hline \multicolumn{4}{|l|}{ C/D Ratio ${ }^{c}$, mean (SD) } \\
\hline Lower & $0.4(0.2)$ & $0.4(0.2)$ & $0.4(0.2)$ \\
\hline Higher & $0.5(0.2)$ & $0.5(0.2)$ & $0.5(0.2)$ \\
\hline \multicolumn{4}{|l|}{ Mean deviation ${ }^{\mathrm{d}}, \mathrm{dB}$, mean (SD) } \\
\hline Lower & $4.7(5.2)$ & $4.8(5.5)$ & $4.7(4.9)$ \\
\hline Higher & $7.8(6.3)$ & $8.0(6.6)$ & $7.5(5.9)$ \\
\hline \multicolumn{4}{|l|}{ Glaucoma-related diagnosis, n (\%) } \\
\hline None & $114(33.1)$ & $52(30.2)$ & $62(36.0)$ \\
\hline Glaucoma & $38(11.0)$ & $17(9.9)$ & $21(12.2)$ \\
\hline Glaucoma suspect & $153(44.5)$ & $80(46.5)$ & $73(42.4)$ \\
\hline Ocular hypertension & $25(7.3)$ & $14(8.1)$ & $11(6.4)$ \\
\hline Fast-tracked at screening (IOP >30 mm Hg) & $14(4.1)$ & $9(5.2)$ & $5(2.9)$ \\
\hline NEI-VFQ composite score ${ }^{\mathrm{e}}$, mean (SD) & $82.2(15.7)$ & $82.1(16.0)$ & $82.3(15.5)$ \\
\hline
\end{tabular}

Abbreviations: C/D ratio, cup-to-disc ratio; dB, decibel; IOP, intraocular pressure; logMAR, logarithm of the minimum angle of resolution; NEI-VFQ, National Eye Institute Visual Function Questionnaire.

${ }^{a}$ Race was unknown for 7 subjects.

${ }^{\mathrm{b}}$ IOP was carried forward from visit 1 for 17 subjects (including 14 fast-tracked subjects).

${ }^{\mathrm{C}} \mathrm{C} / \mathrm{D}$ ratio was not available for 22 subjects (including 14 fast-tracked subjects).

${ }^{\mathrm{d}}$ Mean deviation was not available for 19 subjects (including 14 fast-tracked subjects).

${ }^{\mathrm{e}}$ One subject did not complete the questionnaire; samples sizes vary across subscales. 
Table 2. Summary of Adherence Outcomes, the Philadelphia Telemedicine Glaucoma Detection and Follow-up Study

\begin{tabular}{|c|c|c|c|c|}
\hline Outcome & Usual Care $(n=172) n(\%)$ & Intervention $(n=172) n(\%)$ & All $(n=344)$ RR $(95 \% \mathrm{Cl})$ & $\begin{array}{c}\text { Glaucoma }(n=230) \text { RR } \\
(95 \% \mathrm{Cl})\end{array}$ \\
\hline Attended Visit 3 within 12 months & $67(39.0)$ & $128(74.4)$ & $1.85(1.51-2.28)$ & $1.73(1.37-2.19)$ \\
\hline $\begin{array}{l}\text { Attended any visit at local } \\
\text { ophthalmologist }\end{array}$ & $71(41.3)$ & $134(77.9)$ & $1.83(1.51-2.22)$ & $1.69(1.36-2.09)$ \\
\hline Adherent in Year 1 & $14(8.1)$ & $32(18.6)$ & $2.08(1.14-3.76)$ & $2.30(1.10-4.82)$ \\
\hline Adherent to first follow-up visit (Visit 4) & $39(22.7)$ & $97(56.4)$ & $2.39(1.78-3.22)$ & $2.55(1.79-3.63)$ \\
\hline At least 1 visit in Year 1 & $31(18.0)$ & $71(41.3)$ & $2.18(1.52-3.12)$ & $2.31(1.48-3.62)$ \\
\hline Total visits attended per year & $0.4(0.7)^{\mathrm{a}}$ & $0.9(0.8)^{a}$ & $2.07(1.54-2.78)$ & $1.99(1.44-2.74)$ \\
\hline
\end{tabular}

Abbreviation: RR, relative risk.

${ }^{a}$ Values are mean (SD). 
Table 3. Intervention Effect on Adherence to Follow-up Schedule, by Year of Follow-up, the Philadelphia Telemedicine Glaucoma Detection and Follow-up Study

\begin{tabular}{|c|c|c|c|c|}
\hline Variable & Total & Adherent to Follow-Up, $n$ (\%) & Adjusted Relative Risk (95\% Cl) & $P$ Value $^{\mathrm{a}}$ \\
\hline \multicolumn{5}{|l|}{ Year 1} \\
\hline Usual care & 172 & $14(8.1)$ & Reference & NA \\
\hline Intervention & 172 & $32(18.6)$ & $2.08(1.14-3.76)$ & .02 \\
\hline \multicolumn{5}{|l|}{ Year 2} \\
\hline Usual care & 140 & $9(6.4)$ & Reference & NA \\
\hline Intervention & 129 & $27(20.9)$ & $2.90(1.39-6.02)$ & .004 \\
\hline \multicolumn{5}{|l|}{ Year 3} \\
\hline Usual care & 25 & $1(4.0)$ & Reference & NA \\
\hline Intervention & 25 & $5(20.0)$ & $5.30(0.56-49.95)$ & .15 \\
\hline \multicolumn{5}{|c|}{ Average, year 1-year 2} \\
\hline Usual care & NA & NA & Reference & NA \\
\hline Intervention & NA & NA & $3.92(1.24-12.43)$ & .02 \\
\hline \multicolumn{5}{|c|}{ Glaucoma Subset } \\
\hline \multicolumn{5}{|l|}{ Year 1} \\
\hline Usual care & 120 & $9(7.5)$ & Reference & NA \\
\hline Intervention & 110 & $21(19.1)$ & $2.30(1.10-4.82)$ & .03 \\
\hline \multicolumn{5}{|l|}{ Year 2} \\
\hline Usual care & 98 & $7(7.1)$ & Reference & NA \\
\hline Intervention & 79 & $16(20.3)$ & $2.47(1.05-5.80)$ & .04 \\
\hline \multicolumn{5}{|l|}{ Year 3} \\
\hline Usual care & 19 & $1(5.3)$ & Reference & NA \\
\hline Intervention & 18 & $4(22.2)$ & $4.80(0.56-41.17)$ & .15 \\
\hline \multicolumn{5}{|c|}{ Average, year 2-year 3} \\
\hline Usual care & NA & NA & Reference & NA \\
\hline Intervention & NA & NA & $3.44(1.11-10.63)$ & .03 \\
\hline
\end{tabular}

Abbreviation: NA, not applicable.

${ }^{a} P$ values were calculated by using GEE (generalized estimating equation) Poisson regression models adjusted for Visit 2 recommended follow-up (as a surrogate of disease severity), age at screening, sex, insurance type, and baseline National Eye Institute Visual Function Questionnaire composite score.

The opinions expressed by authors contributing to this journal do not necessarily reflect the opinions of the U.S. Department of Health and Human Services, the Public Health Service, the Centers for Disease Control and Prevention, or the authors' affiliated institutions. 